\title{
Pneumococcal meningitis is promoted by single cocci expressing pilus adhesin RrgA
}

\author{
Federico lovino, Disa L. Hammarlöf, Genevieve Garriss, Sarah Brovall, Priyanka Nannapaneni, and Birgitta Henriques-Normark \\ Department of Microbiology, Tumor and Cell Biology, Karolinska Institutet, and Clinical Microbiology, Karolinska University Hospital, Stockholm, Sweden.
}

\begin{abstract}
Streptococcus pneumoniae (pneumococcus) is the primary cause of bacterial meningitis. Pneumococcal bacteria penetrates the blood-brain barrier (BBB), but the bacterial factors that enable this process are not known. Here, we determined that expression of pneumococcal pilus-1, which includes the pilus adhesin RrgA, promotes bacterial penetration through the BBB in a mouse model. S. pneumoniae that colonized the respiratory epithelium and grew in the bloodstream were chains of variable lengths; however, the pneumococci that entered the brain were division-competent, spherical, single cocci that expressed adhesive RrgA-containing pili. The cell division protein DivIVA, which is required for an ovoid shape, was localized at the poles and septum of pneumococcal chains of ovoid, nonseparated bacteria, but was absent in spherical, single cocci. In the bloodstream, a small percentage of pneumococci appeared as piliated, RrgA-expressing, DivIVA-negative single cocci, suggesting that only a minority of $S$. pneumoniae are poised to cross the BBB. Together, our data indicate that small bacterial cell size, which is signified by the absence of DivIVA, and the presence of an adhesive RrgA-containing pilus-1 mediate pneumococcal passage from the bloodstream through the BBB into the brain to cause lethal meningitis.
\end{abstract}

\section{Introduction}

The most common causes of bacterial meningitis, Streptococcus pneumoniae (pneumococcus), Haemophilus influenzae, and Neisseria meningitidis $(1,2)$, are small in size. However, during nasopharyngeal colonization, $S$. pneumoniae grows in chains of ovoid-shaped cocci that vary in length depending on the completeness of the cell wall cleavage between daughter cells after cell division. Chain formation is believed to allow better adherence to the respiratory epithelium than is seen with small, individual cocci (3). Pneumococcal serotype 6B has been shown to be one of the most frequently found serotypes causing meningitis $(1,2)$. Meningitis is usually caused by bacteria crossing from the bloodstream into the brain through the blood-brain barrier (BBB) (4). Pneumococcal translocation through the BBB is facilitated by receptor-mediated binding to the plasma membrane of endothelial cells (5-7). Previous reports have shown that the surface-anchored neuraminidase A (NanA) protein promotes pneumococcal invasion of brain endothelial cells (8) and that pneumolysin and choline-binding protein A ( $\mathrm{CbpA})$ are important for the development of invasive pneumococcal disease, including meningitis (9). The RlrA pilus (pilus-1) has been shown to increase pathogenicity in animal models and the ability of pneumococci to adhere to host cells (10-13). The $r$ r $A$ pilus islet is composed of 7 genes that encode a transcriptional regulator (RlrA), 3 cell wall surface-anchored family proteins (RrgA, RrgB, and $\operatorname{RrgC})$, and 3 sortases $(10,11)$, resulting in a heteropolymer covalently bound to the cell wall of the bacteria and having a focal distribution $(11,14)$.

Conflict of interest: The authors have declared that no conflict of interest exists Submitted: September 21, 2015; Accepted: May 5, 2016.

Reference information: J Clin Invest. 2016;126(8):2821-2826. doi:10.1172/JCI84705.

\section{Results and Discussion}

Serotype 6B has frequently been isolated from patients with meningitis but has also often been found in carriage specimens (3). Using whole-genome sequencing to explore differences in genetic content among clinical isolates, we selected 5 meningitis and 9 carriage isolates of the same serotype, $6 \mathrm{~B}$, and sequence type, CC138, collected from children (3). All meningitis isolates tested and the carriage isolates, except for carriage isolate BHN460, carried the rlrA pilus islet, which encodes pilus-1. For further studies, we selected the piliated invasive isolate BHN191, the nonpiliated carriage isolate BHN460, and the piliated carriage isolate $\mathrm{BHN} 427$ (3). The presence of the $r l r A$ pilus islet was assessed by genomic sequence alignment. Sequence analysis of the pilus region revealed that BHN191 and BHN427 were identical, except for the insertion of 10 bp (ATACTATACT) in BHN191 upstream of the translational start site for the $r r g A$ pilus adhesin gene (Supplemental Figure 1, A and B, and Supplemental Information; supplemental material available online with this article; doi:10.1172/JCI84705DS1).

Next, we used a bacteremia-derived meningitis mouse model $(6,7,15)$ to study the role played by pilus- 1 in meningitis development. Bacterial counts from brain homogenates demonstrated that mice infected with piliated invasive BHN191 had approximately $80 \%$ more pneumococci in the brain than did mice infected with the nonpiliated carriage isolate BHN460 (Figure 1A). This difference in bacterial load in the brain reflected the score of clinical symptoms. Thus, mice infected with BHN191 showed signs of severe pneumococcal disease, while mice infected with BHN460 showed mild symptoms. Interestingly, mice infected with the piliated carriage isolate BHN427 consistently carried more $(\sim 70 \%)$ bacteria in the brain than did those infected with BHN460, but less ( $30 \%)$ than did those infected 
A

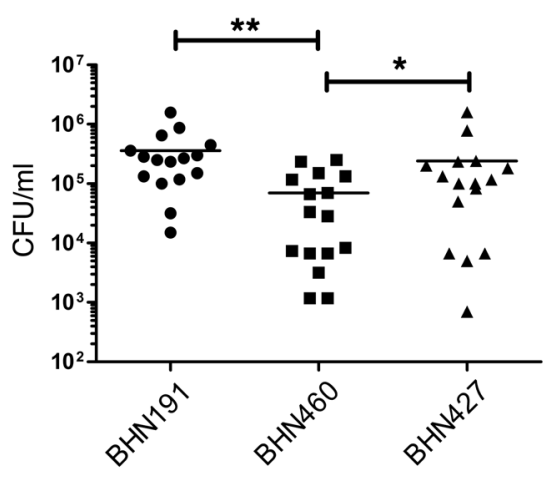

B

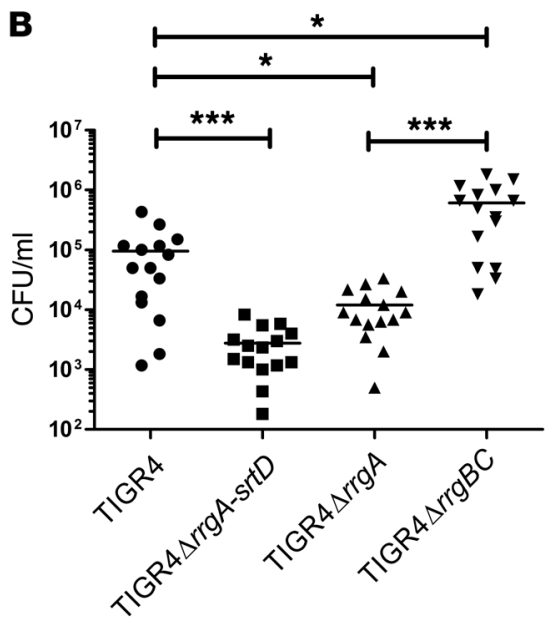

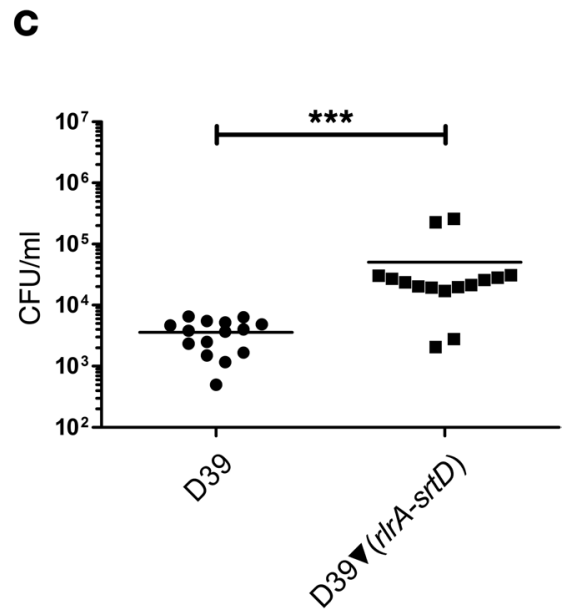

Figure 1. Pilus-1 promotes invasion of S. pneumoniae into the brain. C57BL/6 mice were infected i.v. with (A) serotype 6B isolates, (B) TIGR4 and isogenic pilus mutants, and (C) D39 and piliated D39 $\mathbf{\nabla}$ ( $r$ r $A$-srtD). Graphs show the number of pneumococci found in the brain: each dot represents 1 mouse; black bars show the average values. $n=15$ per group ( 3 experiments with $n=5$ per group). ${ }^{*} P<0.05,{ }^{* *} P<0.01$, and ${ }^{* * *} P<0.001$, by nonparametric ANOVA, followed by Dunn's test (A and B) and by nonparametric, 2-tailed Wilcoxon's rank-sum test (C).

with BHN191 (Figure 1A). To confirm the role played by pilus-1 in pneumococcal meningitis, we challenged mice with the piliated serotype 4 strain TIGR4 or its nonpiliated mutant (TIGR4 $\Delta r r g A-$ $s r t D)(11)$. The number of bacteria in the brain homogenates was more than 10-fold higher for WT TIGR4 than for nonpiliated TIGR4 $\Delta r r g A-s r t D$ (Figure 1B). We next challenged mice with the nonpiliated strain D39 of serotype 2 or its isogenic, complemented piliated strain [D39 $\boldsymbol{\nabla}(r l r A-s r t D)]$ carrying the complete pilus-1 islet from TIGR4 (11). Again, brain homogenates showed a 10-fold higher number of bacteria for mice infected with the piliated as compared with those infected with the nonpiliated strain (Figure 1C). To exclude the possibility that the higher CFU counts observed in mice infected with piliated bacteria were due to the growth phenotype, we also confirmed our data using quantitative imaging analysis (Supplemental Figure 2, A-D). We conclude that pilus-1 promotes pneumococcal invasion into the brain.

RrgA acts as an adhesin to respiratory epithelial cells (12). We therefore investigated whether RrgA promotes entry of pneumococci into the brain across the BBB. Mice were challenged with TIGR4 $\Delta r r g A$, expressing pili but lacking RrgA, or nonpiliated TIGR4 $\triangle r r g B C$ (11), lacking the major stalk protein of the pilus, $\mathrm{RrgB}$, and the minor pilin RrgC, but expressing cell wall-bound RrgA. Bacterial counts in brain homogenates were lower for TIGR4 $\triangle r r g A$ than for WT TIGR4, suggesting that RrgA allows bacterial binding to the $\mathrm{BBB}$ endothelium and thereby promotes the entry of pneumococci into the brain. Interestingly, TIGR $4 \Delta r r g B C$, expressing RrgA in the absence of pili, penetrated the BBB even better than did TIGR4 (Figure 1B).

Microgliosis is a typical process during neuroinflammation, and the resident macrophages of the brain, the microglia, undergo morphological changes, in which their central soma becomes rounder and their long dendrites shorter and thicker (15-17). Immunofluorescence analyses showed that piliated BHN191 and TIGR4 caused severe neuroinflammation, and microglia showed a dramatic change in soma morphology, which appeared to be larger than a normal microglial cell, and almost complete den- drite retraction (Supplemental Figure 3A). Mice infected with nonpiliated BHN460 or TIGR4 $\Delta r r g A-s r t D$ also showed signs of neuroinflammation, but to a lesser extent than did the piliated strains. Brain inflammation was also assessed by quantitative reverse transcriptase PCR for TNF- $\alpha$, IL-6, and IL-1 $\beta$, which are major inflammatory cytokines detected in the brain during bacterial meningitis $(15,18)$, as well as for the microglial marker ionized calcium-binding adapter molecule 1 (Iba-1) $(15,19)$. Piliated BHN191 and TIGR4 showed increased expression levels of TNF- $\alpha$, IL-6, IL-1 $\beta$, and Iba-1, in contrast to nonpiliated BHN460 and TIGR4 $\Delta r r g A-s r t D$ (Supplemental Figure 3, B-E). These results suggest that pilus-1 expression enhances both pneumococcal invasion and inflammation in the brain.

S. pneumoniae are usually found as chains or ovoid diplococci (20). In the bloodstream, in lung homogenates and lung tissue sections, piliated BHN191, TIGR4, and D39 $\mathbf{v}(r \operatorname{lr} A-s r t D)$, and nonpiliated BHN460, TIGR4 $\Delta r r g A-s r t D$, and D39 formed mainly chains (Figure 2, A and B, and Supplemental Figure 4). In the brain tissue, a majority of nonpiliated BHN460, TIGR4 $\Delta r r g A-s r t D$, and D39 were detected as chains (Figure 2, A and B), but surprisingly, piliated BHN191, TIGR4, and D39 $\boldsymbol{\nabla}$ ( $r l r A-s r t D)$ were always detected as single spherical rather than ovoid cocci that only occasionally had a division septum (indicating the formation of a diplococcus) (Figure 2, A and B). The presence of this division septum in some of the piliated pneumococci in the brain indicated that single cocci may be able to grow and divide but that bacterial cell separation has achieved a rapid completion. Since pneumococcal chains could have been disrupted during the centrifugation steps of the brain, we stained brain tissue sections directly for pneumococci. Bacterial chains were again consistently detected for nonpiliated BHN460, TIGR4 $\Delta r r g A-s r t D$, and D39, while single cocci were observed for piliated BHN191, TIGR4, and D39 $\mathbf{v}(r l r A-s r t D)$ (Supplemental Figure 5). The piliated carriage isolate BHN427 and the piliated, nonadhesive mutant strain TIGR $4 \Delta r r g A$ showed a heterogeneous population of single cocci, diplococci, and chains in the brain in both experimental procedures (Figure 2, A and B, 
A
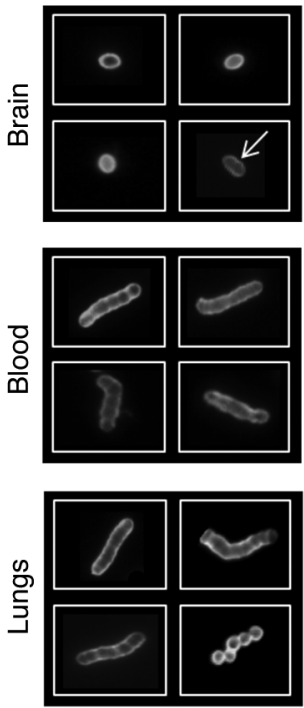

$10 \mu \mathrm{m}$

B

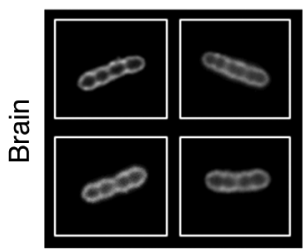

D39 $\boldsymbol{\nabla}$ (rIrA-srtD)
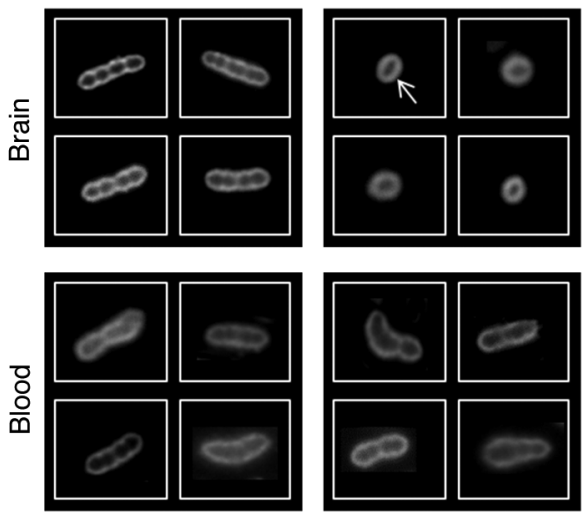

BHN460
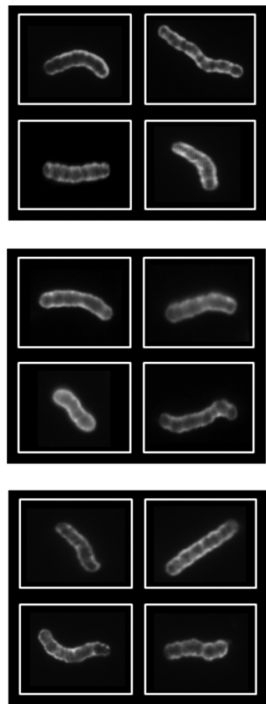

10
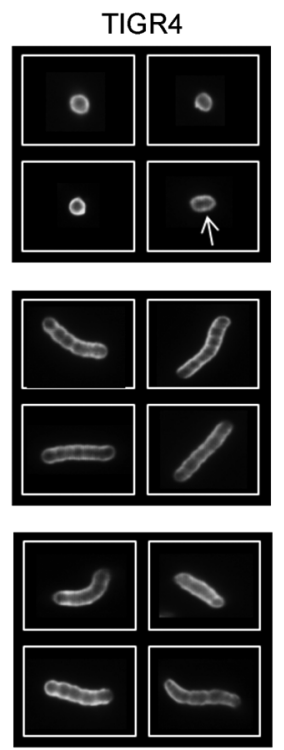

TIGR4 $\triangle r r g B C$
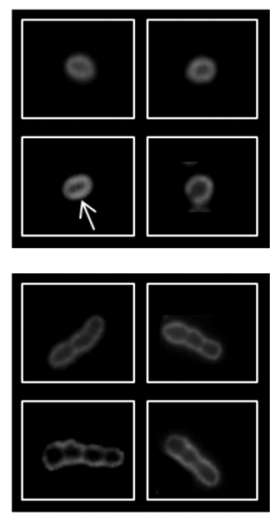

TIGR4 $\triangle r r g A-s r t D$
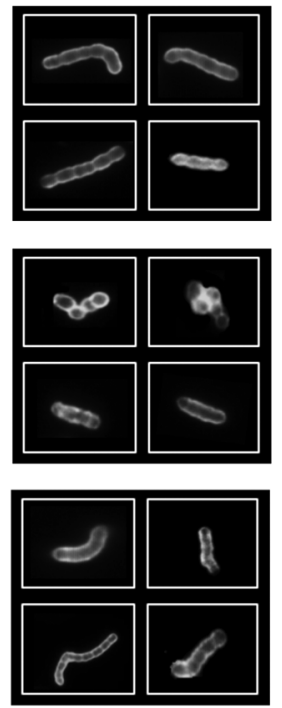

TIGR4 $\Delta r r g A$
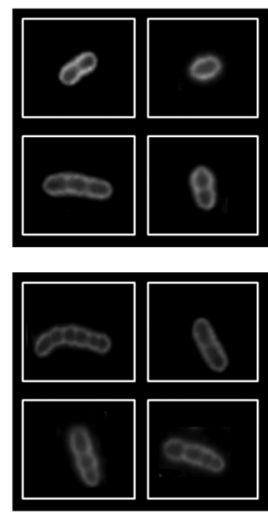

BHN427
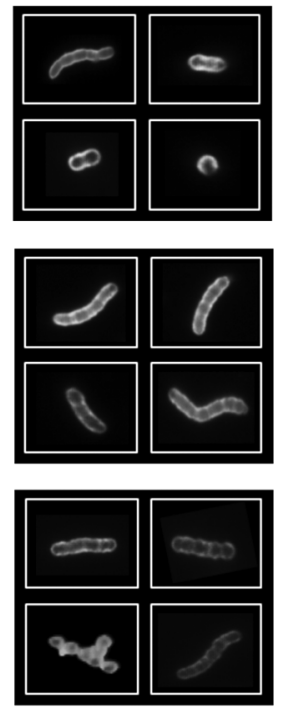

$10 \mu \mathrm{m}$

and Supplemental Figure 5). Intriguingly, the expression levels of pilus-1 proteins in BHN427 were lower than those in BHN191 (Supplemental Figure 6). To show the "active" status of single cocci, we then used high-resolution microscopy to study their expression of FtsZ, the cell division-initiator protein. We found that single cocci express FtsZ at the midzone (21), implying that they are division competent (Supplemental Figure 7).

The pneumococcal protein DivIVA is one of a number of membrane proteins that orchestrate and fine tune cell wall synthesis during septal growth and cell separation and localize to the division septum and to the poles of cocci $(21,22)$. It has been proposed that DivIVA tunes the 2 modes of peptidoglycan (peripheral and septal) synthesis in pneumococci and is responsible for the cell's ovoid shape (22). Deletion of DivIVA hindered cell elongation and resulted in cell shortening and rounding (22). We investigated the expression of DivIVA and the structural pilus proteins RrgA, $\mathrm{RrgB}$, and $\mathrm{RrgC}$ in brain homogenates from mice infected with the 3 clinical isolates of serotype 6B, TIGR4, or TIGR4 $\Delta r r g A-s r t D$. Chains of nonpiliated BHN460 expressed DivIVA, while piliated BHN191, present as single spherical cocci, lacked DivIVA expression (Figure 3A and Supplemental Figure 8). Piliated TIGR4, forming single cocci, did not express DivIVA, while nonpiliated TIGR4 $\Delta r r g A-s r t D$, forming chains, expressed DivIVA. Strain BHN427, expressing fewer pili than did BHN191, showed an intermediate phenotype, in which DivIVA and the pilus-1 proteins were detectable in diplococci and chains; however, DivIVA expression was lower than in nonpiliated BHN460, and only pilus-1 proteins were detected in single cocci (Figure $3 \mathrm{~A}$ and Supplemental Figure 8A). Expression levels of the structural proteins RrgA, RrgB, and $\mathrm{RrgC}$ were higher in BHN191 than in BHN427 in brain tissue (Supplemental Figure 8B), a difference that was also observed in blood, lungs, and Todd-Hewitt broth with $0.5 \%$ yeast extract (THY) medium, but to a considerably lesser extent (Supplemental Figure 9B, Supplemental Figure 10B, and Supplemental Figure 11B). In addition, piliated TIGR4 showed higher expression levels of the 3 pilus components in the brain than in the lungs or blood or under in vitro conditions (Supplemental Figure 8C, Supplemental Figure 9C, Supplemental Figure 10C, and Supplemental Figure 11C).

Among the minority of piliated cocci in the brain showing a division septum, we could distinguish bacteria in the early and late stages of cell division by high-resolution microscopy (Supplemental Figure 12, A and B). Even though most single cocci did not express 
A
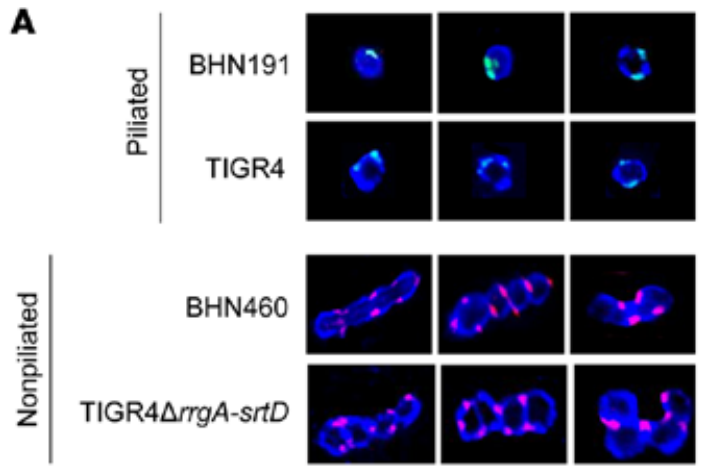

$\mathrm{BHN} 427$
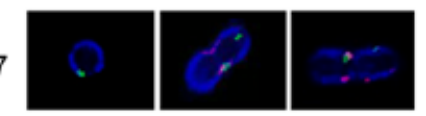

$5 \mu \mathrm{m}$
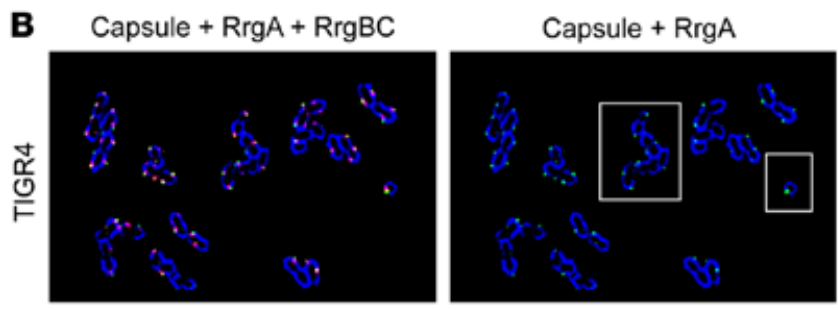

Enhanced magnification
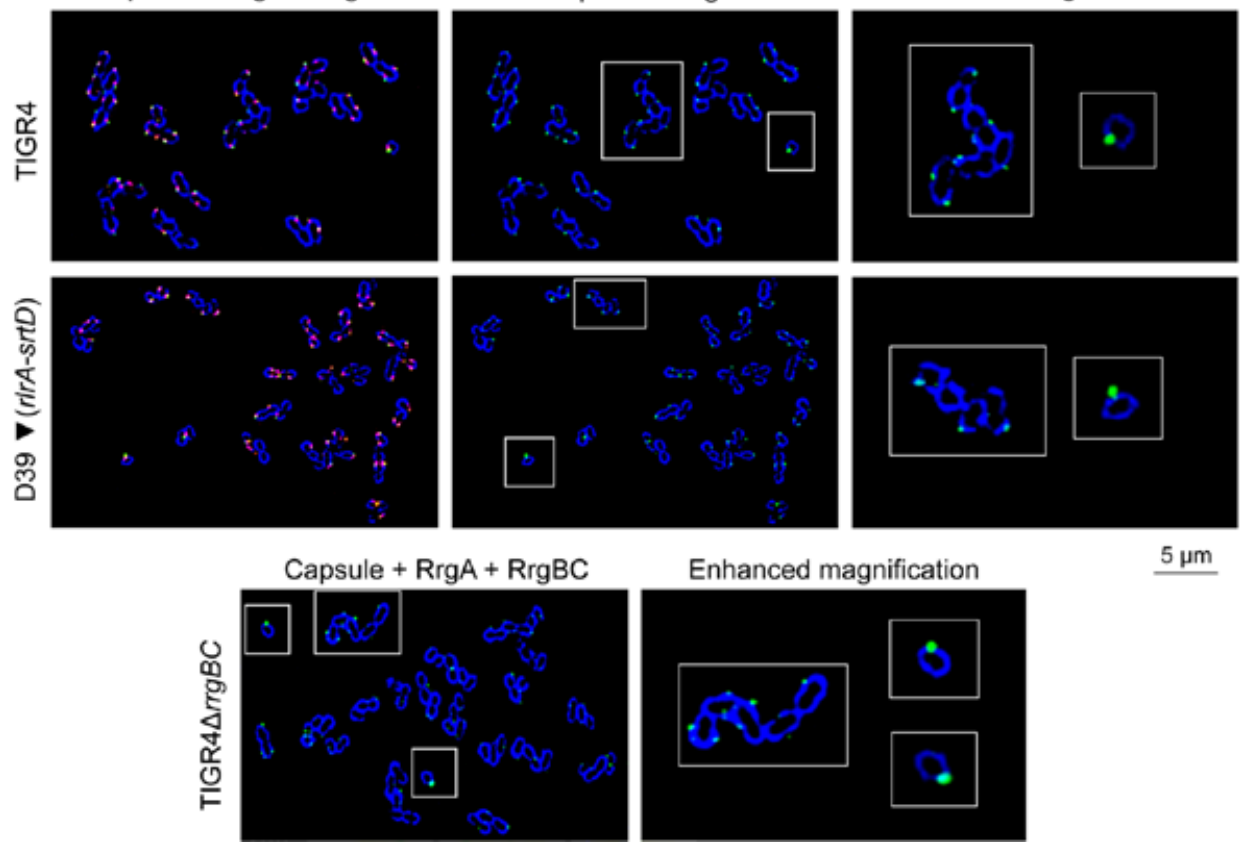

Enhanced magnification

$\underline{5 \mu \mathrm{m}}$

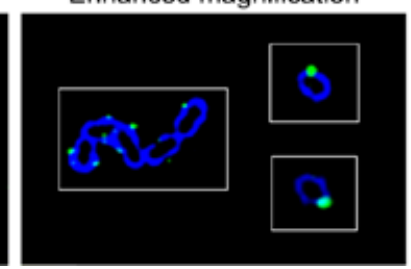

DivIVA in the brain, bacteria caught in the division process were DivIVA positive. In particular, at the early stages of cell division, when bacteria had a round shape, with the formation of a division septum, DivIVA was expressed at the poles of the cells (Supplemental Figure 12A). During cell division, the bacterial cell was more elongated and more closely resembled a diplococcus with 2 nearly formed daughter cells. At this stage, the DivIVA signal was severely decreased (Supplemental Figure 12B). These findings strongly suggest that the DivIVA protein is not always absent in piliated bacteria during brain invasion but is instead present for only a limited time while single cocci are dividing. This very limited expression of DivIVA only in dividing cocci was not enough to be detected by Western blotting (Supplemental Figure 8). When a diplococcus was almost formed, the DivIVA signal had almost disappeared, suggesting that DivIVA undergoes a rapid degradation. All 5 strains formed chains in blood and lungs and under in vitro conditions, and DivIVA was expressed (Supplemental Figure 9A, Supplemental Figure 10A, and Supplemental Figure 11A). Consistently, nonpiliated BHN460
Figure 3. In the blood, piliated and RrgA-expressing single cocci, typified by the absence of DivIVA, express more RrgA than do chains and are more prone to penetration of the BBB. (A) Immunofluorescence staining showing capsule (blue), pilus-1 (green), and DivIVA (red) of pneumococci in brain homogenates. For each strain, 3 mice were analyzed (approximately 300 images per mouse were taken). (B) Immunofluorescence staining of RrgA-expressing pneumococci in blood samples showing capsule (blue), RrgA (green), and RrgBC (red). "Enhanced magnification" panels show the parts within the white-outlined areas at higher magnification (original magnification, $\times 5$ ). One representative image per strain is shown ( $\times 100$ objective); in total, approximately 900 bacteria per strain were imaged. and TIGR4 $\Delta r r g A-s r t D$ showed higher expression levels of DivIVA compared with levels in piliated BHN191, BHN427, and TIGR4 (Supplemental Figure 9, B and C; Supplemental Figure 10, B and C; Supplemental Figure 11, B and C).

To determine whether single cocci exist outside the brain, we investigated a large number of bacteria for all 5 strains growing in blood. The piliated strains BHN191, BHN427, TIGR4, and D39 $\boldsymbol{\nabla}(r l r A-s r t D)$ formed chains of various lengths, but, notably in the blood, a very small percentage $(<5 \%)$ appeared as single, spherical cocci (Supplemental Figure 13A) that did not express DivIVA (Supplemental Figure 13B). The nonpiliated strains BHN460, TIGR4 $\Delta r r g A-s r t D$, and D39 formed chains that expressed DivIVA, but no single cocci were detected among 1,000 bacteria studied (data not shown). Interestingly, the nonpiliated TIGR4 mutant expressing the adhesin RrgA directly linked to the cell wall (TIGR4 $\triangle r r g B C$ ) also produced small, single cocci in the bloodstream that likewise did not express DivIVA (Supplemental Figure 13B). In all cases, single cocci in the blood expressed pilin pro- 
teins (Figure 3B and Supplemental Figure 13C). Thus, single cocci appear in the blood as a minor cell population when pneumococci express at least 1 pilin protein anchored to the cell wall. Notably, through high-resolution microscopy, we observed that, among all strains expressing the adhesin RrgA [TIGR4, TIGR4 $\triangle r r g B C$, and D39 $\boldsymbol{\nabla}(r l r A-s r t D)]$, single cocci showed higher expression levels of RrgA than did chains (Figure 3B). TIGR4 $\Delta r r g A$ and TIGR4 $\triangle r r g B C$ were both present as single cocci in the blood, but invasion of nonadhesive TIGR $4 \Delta r g A$ into the brain was almost 10 times lower than for adhesive TIGR4 $\triangle r r g B C$ (Figure 1B). Among the TIGR4 $\triangle r r g A$ cells that entered the brain, a mixed population of single cocci, diplococci, and chains was observed in contrast to that observed for TIGR $4 \Delta r g B C$ cells (Figure 2, B and C). Thus, high expression levels of the pilus adhesin RrgA probably promote the ability of single, spherical cocci to adhere to the vascular endothelium in the $\mathrm{BBB}$, a prerequisite for subsequent invasion into the brain.

In the bloodstream, single cocci only appeared in pneumococcal strains able to express pili or at least 1 of the 3 pilus proteins. The underlying mechanism for this single coccus minority in a predominantly chain-forming cell population might involve cell wall alterations at the site of cell separation due to pilus/pilin anchoring to the cell wall. Our results suggest that the small minority of adhesive, single cocci in the bloodstream are more prone to BBB penetration than are chains or nonadhesive, single cocci (Supplemental Figure 14). Once they have invaded, single spherical cocci appear to be division competent in the brain, as evidenced by the expression of FtsZ (Supplemental Figure 7).

Taken together, our data suggest that pneumococcal expression of pili in the bloodstream promotes the formation of single cocci that, when expressing the pilus adhesin RrgA, have a greater ability to penetrate the BBB. Even though the fraction of small cocci in the circulation is low, they will continuously seed the brain endothelium during the infection with invasion-competent, adhesive bacteria. It is worth noting that the other 2 major bacterial species known to cause meningitis, $H$. influenzae and N. meningitides, are small-sized bacteria. This is also true for $S$. pneumoniae, an organism in which each individual bacterium only measures approximately $1 \mu \mathrm{m}$ compared with a chain, which can be longer than $10 \mu \mathrm{m}$. It has previously been demonstrated that particles of small molecular size penetrate the BBB more easily than do larger molecules $(23,24)$.
Size constraints may therefore explain why a single coccus can penetrate the $\mathrm{BBB}$ more easily than a long chain of unseparated bacteria, even when both adhere to the BBB endothelium.

\section{Methods}

A complete description of all methods is provided in the Supplemental Methods.

Statistics. For multiple comparisons, nonparametric ANOVA was used to assess the presence of between-groups differences, followed by Dunn's test for pairwise comparisons. For 2-group comparisons, the nonparametric 2-tailed Wilcoxon's rank-sum test (also known as the Mann-Whitney $U$ test) was used. A $P$ value of less than 0.05 was considered statistically significant.

Study approval. All animal experiments were approved by the Stockholm Norra djurförsöksetiska nämnd ethics committee (Stockholm, Sweden).

\section{Author contributions}

FI and BHN conceived and designed the study experiments and analyzed data. FI, DLH, GG, PN, and SB performed experiments and analyzed data. FI and BHN wrote the manuscript. DLH, GG, $\mathrm{PN}$, and SB contributed to the writing of the manuscript.

\section{Acknowledgments}

We thank Orietta Massidda for providing anti-DivIVA antiserum and anti-FtsZ Ab; Katalin Benedek for the cryostat cutting of tissue sections; Ilias Galanis for help with statistical analyses; Kenth Andersson for technical assistance during mouse experiments; and Staffan Normark for scientific discussions. This work was supported by grants from the Knut and Alice Wallenberg Foundation; the Swedish Research Council; the Swedish Foundation for Strategic Research (SSF); an Anslag till forskning (ALF) grant from the Stockholm County Council; and a Marie Curie Label-Free Particle Sorting (LAPASO) project grant, funded by the European Union.

Address correspondence to: Birgitta Henriques-Normark, Karolinska Institutet, Nobelsväg 16, SE-171 77 Stockholm, Sweden. Phone: 46851771215; E-mail: birgitta.henriques@ki.se.

Disa L. Hammarlöf's present address is: Department of Cell and Molecular Biology, Uppsala Universitet, Uppsala, Sweden.
1. Brouwer MC, Tunkel AR, van de Beek D. Epidemiology, diagnosis, and antimicrobial treatment of acute bacterial meningitis. Clin Microbiol Rev. 2010;23(3):467-492.

2. Browall S, et al. Intraclonal variations among Streptococcus pneumoniae isolates influence the likelihood of invasive disease in children. J Infect Dis. 2014;209(3):377-388.

3. Weiser JN. The battle with the host over microbial size. Curr Opin Microbiol. 2013;16(1):59-62.

4. Mook-Kanamori BB, Geldhoff M, van der Poll T, van de Beek D. Pathogenesis and pathophysiology of pneumococcal meningitis. Clin Microbiol Rev. 2011;24(3):557-591.

5. Orihuela CJ, et al. Laminin receptor initiates bacterial contact with the blood brain barrier in experimental meningitis models. JClin Invest.
2009;119(6):1638-1646.

6. Iovino F, Molema G, Bijlsma JJ. Streptococcus pneumoniae Interacts with pIgR expressed by the brain microvascular endothelium but does not co-localize with PAF receptor. PLoS One. 2014;9(5):e97914.

7. Iovino F, Molema G, Bijlsma JJ. Platelet endothelial cell adhesion molecule-1, a putative receptor for the adhesion of Streptococcus pneumoniae to the vascular endothelium of the blood-brain barrier. Infect Immun. 2014;82(9):3555-3566.

8. Uchiyama S, et al. The surface-anchored NanA protein promotes pneumococcal brain endothelial cell invasion. JExp Med. 2009;206(9):1845-1852.

9. Mann B, et al. Broadly protective protein-based pneumococcal vaccine composed of pneumolysin toxoid-CbpA peptide recombinant fusion protein.
JInfect Dis. 2014;209(7):1116-1125.

10. Barocchi MA, et al. A pneumococcal pilus influences virulence and host inflammatory responses. Proc Natl Acad Sci U S A. 2006;103(8):2857-2862.

11. Fälker S, et al. Sortase-mediated assembly and surface topology of adhesive pneumococcal pili. Mol Microbiol. 2008;70(3):595-607.

12. Nelson AL, et al. RrgA is a pilus-associated adhesin in Streptococcus pneumoniae. Mol Microbiol. 2007;66(2):329-340.

13. Hava DL, Camilli A. Large-scale identification of serotype 4 Streptococcus pneumoniae virulence factors. Mol Microbiol. 2002;45(5):1389-1406.

14. Kandaswamy K, et al. Focal targeting by human $\beta$-defensin 2 disrupts localized virulence factor assembly sites in Enterococcus faecalis. Proc Natl 
Acad Sci U S A. 2013;110(50):20230-20235.

15. Iovino F, Orihuela CJ, Moorlag HE, Molema G, Bijlsma JJ. Interactions between blood-borne Streptococcus pneumoniae and the blood-brain barrier preceding meningitis. PLoS One. 2013;8(7):e68408.

16. Zhang D, Hu X, Qian L, O'Callaghan JP, Hong JS. Astrogliosis in CNS pathologies: is there a role for microglia? Mol Neurobiol. 2010;41(2-3):232-241.

17. Wen YR, Tan PH, Cheng JK, Liu YC, Ji RR.

Microglia: a promising target for treating neuropathic and postoperative pain, and morphine tolerance. J Formos Med Assoc. 2011;110(8):487-494.

18. Fassbender K, Ries S, Schminke U, Schneider S,
Hennerici M. Inflammatory cytokines in CSF in bacterial meningitis: association with altered blood flow velocities in basal cerebral arteries. J Neurol Neurosurg Psychiatr. 1996;61(1):57-61.

19. Ito D, Tanaka K, Suzuki S, Dembo T, Fukuuchi Y. Enhanced expression of Iba1, ionized calcium-binding adapter molecule 1 , after transient focal cerebral ischemia in rat brain. Stroke. 2001;32(5):1208-1215.

20. Tomasz A, Jamieson JD, Ottolenghi E. The fine structure of Diplococcus pneumoniae.JCell Biol. 1964;22:453-467.

21. Fadda D, et al. Streptococcus pneumoniae DivIVA: localization and interactions in a MinCD-free context. JBacteriol. 2007;189(4):1288-1298.

22. Fleurie A, et al. Interplay of the serine/threonine-kinase StkP and the paralogs DivIVA and GpsB in pneumococcal cell elongation and division. PLoS Genet. 2014;10(4):e1004275.

23. Banks WA. Characteristics of compounds that cross the blood-brain barrier. BMC Neurol. 2009;9(suppl 1):S3.

24. Hanada S, Fujioka K, Inoue Y, Kanaya F, Manome Y, Yamamoto K. Cell-based in vitro blood-brain barrier model can rapidly evaluate nanoparticles' brain permeability in association with particle size and surface modification. Int J Mol Sci. 2014;15(2):1812-1825. 